\title{
Correspondence
}

\section{Influence of Extrinsic Contribution on the Macroscopic Properties of Hard and Soft Lead Zirconate Titanate Ceramics}

\author{
Diego A. Ochoa, Jose E. García, Rafel Pérez, \\ and Alfons Albareda, Member, IEEE
}

\begin{abstract}
In this work, the contribution of the extrinsic effect to the macroscopic properties in soft and hard lead zirconate titanate ceramics is directly evaluated. Close to the room temperature, poled hard ceramics show an anomalous behavior, which is notably different from that of soft ceramics, not only in dielectric but also in piezoelectric and elastic responses. Hence, at room temperature their properties are thermally stable and the losses are unusually low. It is suggested that two mechanisms are present, with one mechanism inhibiting the other.
\end{abstract}

\section{INTRODUCTION}

$\mathrm{T}$ HE use of devices employing piezoelectric ceramics is still expanding, and they form part of many high technology devices. Among the most useful piezoelectric materials, we find lead zirconate titanate based ceramics (PZT). They are probably the most widely used piezoelectric materials because of their high piezoelectric coefficient value, in addition to their low mechanical and dielectric losses [1], [2].

The elastic, piezoelectric, and dielectric responses in these materials are due to 2 different kinds of phenomena. The first, called the intrinsic effect, is related to the polarization of the elemental cell and would be the sole response if the material was in a single domain state. The second, known as the extrinsic effect, includes all the phenomena different from the intrinsic effect, although it is commonly accepted in the literature that the major contribution to the extrinsic effect comes from the motion of the domain walls and their interaction with the lattice defects [3]-[5]. Zhang et al. have shown that the intrinsic contribution to the material response can be considered nearly constant with the temperature. Moreover, these authors make it clear that the extrinsic effect depends strongly on temperature [3]. The extrinsic effect consists in the motion of some elements (i.e., the domain walls) whose mobility tends to be null at absolute temperature $(T=0 \mathrm{~K})$. Therefore, at very low temperature the response of the material can be due exclusively to the intrinsic effect. Nevertheless, the extrinsic effect provides a major contribution to dielectric and piezoelectric response

Manuscript received May 13, 2008; accepted July 21, 2008. This work was supported by Project MAT2007-63445 of the Spanish MEC.

The authors are with Department of Applied Physics, Universitat Politècnica de Catalunya, Barcelona, Spain (e-mail: jose@fa.upc.edu).

Digital Object Identifier 10.1109/TUFFC.2008.990 at room temperature and is therefore responsible for the excellent properties of PZT [3].

The macroscopic properties of ceramics based on $\mathrm{Pb}\left(\mathrm{Zr}_{1-x} \mathrm{Ti}_{x}\right) \mathrm{O}_{3}$ mainly depend on the domain structure, which is determined by the $\mathrm{Zr} / \mathrm{Ti}$ ratio. Close to $x=0.47$ $(\mathrm{Zr} / \mathrm{Ti}=53 / 47)$, PZT shows a morphotropic phase boundary (MPB), and in that region the macroscopic properties become exceptional [1]. In the attempt to improve the PZT properties for specific applications, impurities are usually added as substitution ions. At room temperature, the macroscopic properties of PZT are substantially modified by the addition of low amounts of these ions. As a result of classification of PZT as a function of their physical properties, which is very often used in the literature, they are classified into 2 groups known as hard and soft PZT. The hard ones are those to which acceptor impurities have been added, usually denoted as hardeners, such as $\mathrm{Fe}^{3+}$. These kinds of ions enter at $\mathrm{Zr}^{4+}$ or $\mathrm{Ti}^{4+}$ sites by creating oxygen vacancies and forming the so-called complex defects [6], [7]. At room temperature, this type of defect acts as a pinning center by lowering the mobility of the domain walls [7], resulting in a fall on the observed values of piezoelectric, elastic, and dielectric coefficients, accompanied by a drop in the material losses. Soft PZT, on the other hand, have been doped by donor impurities, known as softeners, such as $\mathrm{Nb}^{5+}$. These ions substitute $\mathrm{Zr}^{4+}$ or $\mathrm{Ti}^{4+}$ ions by forming lead vacancies [1] and by removing oxygen vacancies [8], thus producing a relaxation of the local stresses of the lattice while minimizing the creation of complex defects associated with the oxygen vacancies.

The macroscopic properties of PZT-based ceramics are mostly due to the contribution of the extrinsic effect, which in turn strongly depends on the addition of one or another type of impurity. Despite previous attempts, the direct relation between the extrinsic contribution and macroscopic properties still remains to be established in these materials. This is the reason that the contribution of the extrinsic effect to the elastic, piezoelectric, and dielectric coefficients, as well as to the elastic and dielectric losses are evaluated in this work. We give an interpretation of the anomalous behavior of the extrinsic response in acceptor-doped PZT as the origin of the differences between the macroscopic properties of soft and hard PZT at room temperature.

\section{EXPERIMENTAL}

\section{A. Tested Materials}

A conventional oxide mixing process was used to prepare $\mathrm{Pb}\left(\mathrm{Zr}_{0.6} \mathrm{Ti}_{0.4}\right) \mathrm{O}_{3}$ ceramics doped with 2 wt.\% of $\mathrm{Nb}_{2} \mathrm{O}_{5}$ (donor-doped) or $\mathrm{Fe}_{2} \mathrm{O}_{3}$ (acceptor-doped), hereafter referred to as PNZT and PFZT, respectively. Precur- 
sors were mixed in a ball mill for $2 \mathrm{~h}$, dried at $80^{\circ} \mathrm{C}$, and calcined at $850^{\circ} \mathrm{C}$ for $3.5 \mathrm{~h}$. After being pressed, the samples were sintered at $1200^{\circ} \mathrm{C}(\mathrm{PFZT})$ and $1245^{\circ} \mathrm{C}(\mathrm{PNZT})$ in a saturated $\mathrm{PbO}$ atmosphere. Scanning electron micrographs showed an average grain size between 3.0 and $3.5 \mu \mathrm{m}$. X-ray diffraction analysis revealed the presence of the rhombohedral phase. Neither spurious phases nor contaminants were detected. For each material, a bar of dimensions $a=15 \mathrm{~mm}$ long, $b=5 \mathrm{~mm}$ wide and $c=$ $1 \mathrm{~mm}$ thick was cut and poled along the $c$-direction.

The best properties of PZT can undoubtedly be obtained with compositions close to MPB. However, the complexity of this region makes the a priori analysis of the experimental results very difficult. A recent study on PZT proves that for both dopants the observed dependence on the temperature of the extrinsic response does not depend on the crystallographic phase [9]. Thus, to avoid interpretation difficulties of materials in MPB, the study has been carried out on samples belonging to the pure rhombohedral phase.

\section{B. Measurement Method}

To evaluate the contribution of the extrinsic effect, a scan of temperature from $20 \mathrm{~K}$ to $400 \mathrm{~K}$, with heating rate $0.5 \mathrm{~K} / \mathrm{min}$, was performed by using a closed loop cold finger cryogenic system. The measurements were taken by an impedance analyzer either at resonance or at low frequency. The amplitude of the voltage applied to the sample was $100 \mathrm{mV}$. Hence, the electric field is not higher than $10^{-3} \mathrm{kV} / \mathrm{cm}$ at low frequency, and the electrical current is lower than $10 \mathrm{~mA}$ even at resonance. These values are low enough so that the extrinsic effect is not perturbed by the nonlinear behavior that appears in PZT when high electrical or mechanical fields are applied [5]. Resonance and antiresonance frequencies of the main vibration extensional mode were measured at each temperature. The capacitance $C^{P}$, which corresponds to the sample capacitance when the motional admittance is null, was measured at a frequency that was twice the value of the resonant frequency. Moreover, real and imaginary parts of the permittivity $\left(\varepsilon_{33}^{\prime}\right.$ and $\varepsilon_{33}^{\prime \prime}$, respectively) were measured at $1 \mathrm{kHz}$.

To obtain the coefficients without using the equivalent circuit approximations, we take the admittance exact solutions for an extensional resonance of a lateral field slim bar [10]. For this geometry, the expression of the admittance is

$$
Y=j \omega \frac{l w}{t} \varepsilon_{33}^{T}\left[k_{31}^{2} \frac{\tan \left(\gamma \alpha^{E}\right)}{\gamma \alpha^{E}}+1-k_{31}^{2}\right]
$$

where $l, w$, and $t$ are the length, width, and thickness of the sample, respectively; $\varepsilon_{33}$ is the stress constant permittivity; $k_{31}$ is the electromechanical coupling factor, and $\omega$ is the angular frequency. The term $\gamma$ is proportional to the frequency and is equal to

$$
\gamma=\frac{\omega l}{2}
$$

while the slowness $\alpha^{E}$ is equal to

$$
\alpha^{E}=\sqrt{\rho s_{11}^{E}}
$$

where $\rho$ is the sample density and $s_{11}^{E}$ is the constant electric field compliance.

From the resonance condition $(Y \rightarrow \infty)$ applied to $(1)$ for the main vibration mode, at resonant frequency $f_{r}$ it can be stated that $\gamma_{r} \alpha^{E}=\pi / 2$, and hence the elastic compliance can be determined:

$$
s_{11}^{E}=\frac{1}{4 \rho l^{2} f_{r}^{2}} .
$$

The coupling coefficient can be obtained by applying the antiresonance condition in (1), where the admittance is null $(Y \rightarrow 0)$ :

$$
\frac{1}{k_{31}^{2}}=1-\frac{\tan \left(\gamma_{a} \alpha^{E}\right)}{\gamma_{a} \alpha^{E}}
$$

where

$$
\gamma_{a} \alpha^{E}=\frac{\pi}{2} \frac{f_{a}}{f_{r}}
$$

The capacitance $C^{P}$ allows us to obtain the permittivity $\varepsilon_{33}^{T}$ :

$$
\varepsilon_{33}^{T}=\frac{t}{w l} \frac{1}{1-k_{31}^{2}} C^{P}
$$

Finally, the piezoelectric constant $d_{31}$ is directly obtained from

$$
d_{31}^{2}=k_{31}^{2} \varepsilon_{33}^{T} s_{11}^{E}
$$

\section{Results And Discussion}

In Fig. 1, one may observe the dependence on the temperature of the dielectric constant, $\varepsilon^{\prime} / \varepsilon_{0}$, and dielectric losses, $\varepsilon^{\prime \prime} / \varepsilon_{0}$, measured at $1 \mathrm{kHz}$, both on donor and acceptor doped PZT samples (PNZT and PFZT, respectively). It is necessary to point out that the permittivity obtained from (7) does not show any significant difference from the measure taken at $1 \mathrm{kHz}$.

The PNZT dielectric constant follows the expected behavior. A monotonous increment of the extrinsic contribution is observed, so that at room temperature the dielectric 


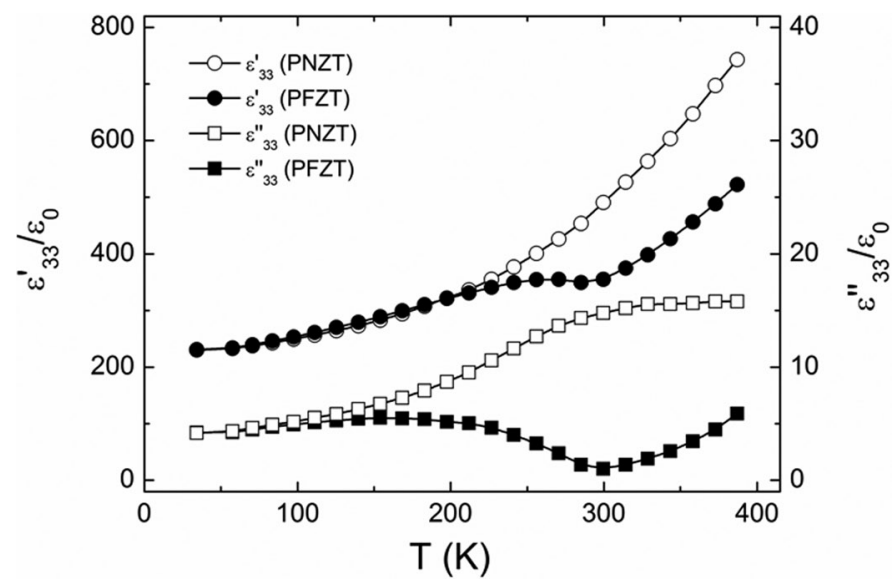

Fig. 1. Temperature dependence of dielectric constant $\varepsilon^{\prime} / \varepsilon_{0}$ and dielectric losses $\varepsilon^{\prime \prime} / \varepsilon_{0}$ in donor-doped (PNZT) and acceptor-doped (PFZT) PZT ceramics.

constant is slightly more than double that obtained at low temperature. On the other hand, in PFZT an anomalous behavior is observed on $\varepsilon^{\prime} / \varepsilon_{0}$ in the range between $200 \mathrm{~K}$ and room temperature such that the dielectric constant has a relative thermal stability around room temperature, and its dielectric constant value is significantly lower than PNZT at room temperature. Note that both the behavior and the value of the dielectric constant are very similar, under $200 \mathrm{~K}$, independently of the type of doping, as has already been observed by other authors [3]. By extrapolating the curves of $\varepsilon^{\prime} / \varepsilon_{0}$, the value of the intrinsic dielectric constant of PZT at rhombohedral phase is 210. Values of 350 have been reported for PZT at the morphotropic phase boundary [3], [9].

By analyzing the dielectric losses, we can observe that in $\mathrm{PNZT} \varepsilon^{\prime \prime} / \varepsilon_{0}$ rises when the temperature is increased, so that its value at room temperature is 3 times the value at very low temperature. Furthermore, PFZT losses show a similar anomalous behavior to permittivity $\varepsilon^{\prime} / \varepsilon_{0}$. In this case, the anomaly appears as a decrease of the dielectric losses, so that it shows a minimum at room temperature, whose value is 4 times less than at low temperature. At low temperature, neither the dielectric constant nor the dielectric losses depend on the ceramic dopants, and the value of $\varepsilon^{\prime \prime} / \varepsilon_{0}$ is equal to 4 units.

In Fig. 2, one may see the dependence on the temperature of the elastic compliance $s_{11} E$ and of the mechanical losses tangent $\tan \delta_{m}$ for both PNZT and PFZT. From zero to room temperature, PNZT compliance increases by $8 \%$, and it always maintains a regular rising behavior. On the other hand, PFZT compliance shows an unexpected behavior similar to that of the dielectric constant. From $200 \mathrm{~K}, s_{11} E$ begins to fall until it reaches a minimum at room temperature, where compliance attains the same value as at low temperature. Similarly to the dielectric behavior, the compliance of both samples are nearly equal at this temperature (around $9.8 \times 10^{-12} \mathrm{~m}^{2} / \mathrm{N}$ ); thus the intrinsic value of the compliance does not depend on the dopants. The plot of PNZT mechanical losses shows a

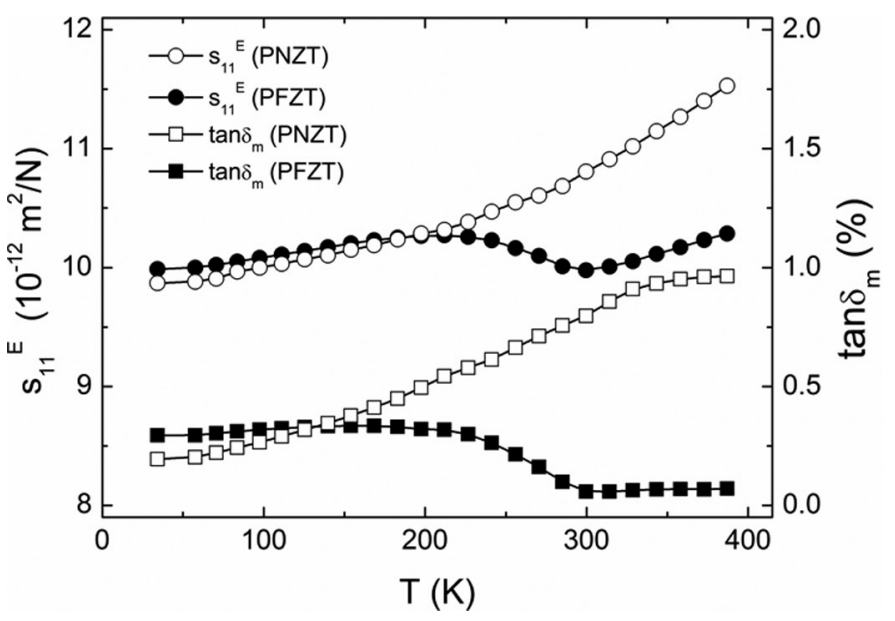

Fig. 2. Temperature dependence of compliance $s_{11} E$ and mechanical losses $\tan \delta_{m}$ in donor-doped (PNZT) and acceptor-doped (PFZT) PZT ceramics.

regular increase with the temperature, reaching a value 4 times greater at room temperature than at low temperature. However, in PFZT the losses begin to decrease at $200 \mathrm{~K}$, and they reach a minimum near room temperature that is 5 times less than at low temperature. The value of the mechanical losses tangent at low temperature is $0.25 \%$ in both materials.

Fig. 3 shows the dependence on temperature of the piezoelectric constant $d_{31}$ for both PNZT and PFZT. In PNZT, the piezoelectric constant rises regularly from 30 $p \mathrm{C} / \mathrm{N}$ at low temperature to double this value at room temperature. However, similar to the former coefficients, we observe that PFZT shows an anomaly near room temperature. As a consequence, around room temperature, $d_{31}$ is thermally stable. This advantageous thermal stability produced by the existence of the anomaly is accompanied by a considerable drop in PFZT response with respect to $\mathrm{PNZT}$.

It is important to point out that unlike observed dielectric and mechanical responses, there is a difference in the value of the piezoelectric constant of the 2 samples at low temperature, a fact previously reported in reference to the constants $d_{31}$ and $d_{33}$ and attributed to the differences in the value of the remnant polarization of those materials [3]. It is well known that between hard and soft PZT there are noticeable differences in remnant polarization throughout the studied temperature range [1], [3].

Fig. 4 shows one of the possible plots in which the anomaly appears very clearly. In this case, compliance is plotted as a function of the dielectric constant for different temperatures. While at low temperature, soft and hard ceramics behave in a similar way, whereas at higher temperatures they have a behavior qualitatively similar, but with different values. Around a certain temperature a sudden change is produced from one behavior to another, so the anomaly has a very characteristic change in such a plot. Other plots, such as $d_{31}\left(s_{11}\right)$ or the imaginary versus real part of dielectric constant, are also characteristic. 


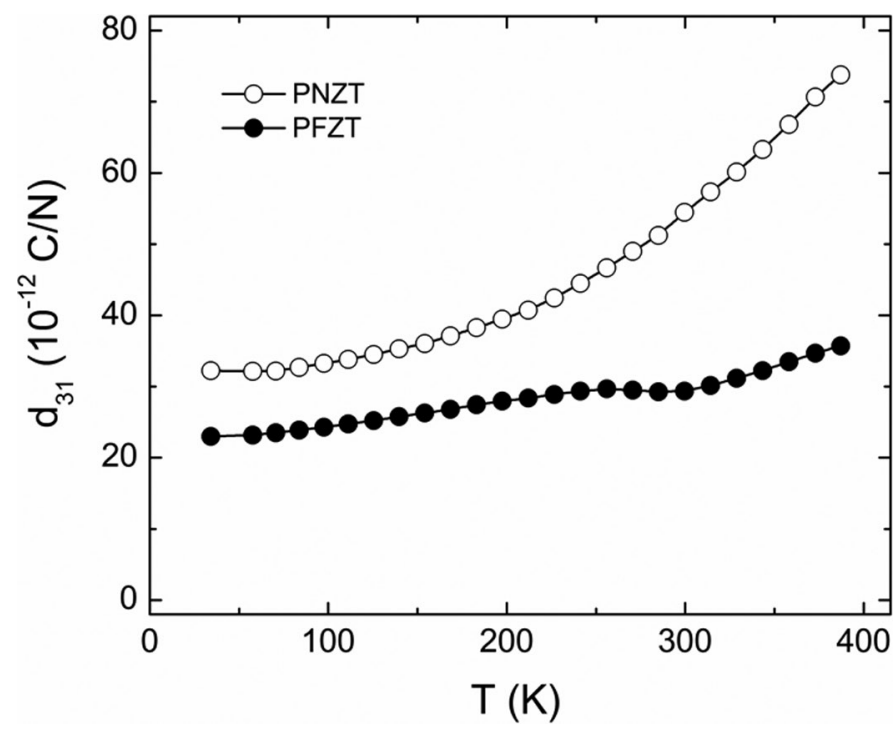

Fig. 3. Temperature dependence of piezoelectric constant $d_{31}$ in donordoped (PNZT) and acceptor-doped (PFZT) PZT ceramics.

\section{COnClusions}

The results presented in this work show that there is a strong dependence on the temperature of the macroscopic properties, not only dielectric $\left(\varepsilon_{33}^{\prime}\right)$, elastic $\left(s_{11}{ }^{E}\right)$, and piezoelectric $\left(d_{31}\right)$ constants, but also dielectric $\left(\varepsilon_{33}{ }^{\prime \prime}\right)$ and mechanical $\left(\tan \delta_{m}\right)$ losses. Furthermore, noticeable differences are seen both quantitatively and qualitatively as regards dependence on the temperature of those macroscopic properties, depending on the type of dopants. It is verified that these differences are related only with the variation of the extrinsic contribution with temperature.

In spite of the different dopants used, the dependence of dielectric and elastic coefficients with temperature is the same at low temperatures. This fact can be explained by taking into account that the domain walls are frozen (due to the mobility drop) at low temperatures, so that the intrinsic contribution is predominant. The intrinsic contribution, which depends on the crystallographic phase, will not depend on the dopants providing they do not alter the lattice characteristics. For this reason, it is possible to state that the differences observed in the macroscopic properties of soft and hard PZT are due entirely to the differences in the extrinsic response of the materials, which in turn depends on the type of dopants included. As regards the piezoelectric behavior, the difference between the intrinsic response of both materials can be explained by the expected differences of their remnant polarization. Nevertheless, it can be remarked that both samples follow the same tendency.

The extrinsic response of hard PZT shows an unexpected behavior over a temperature range close to the room temperature. Anomalous dielectric behavior in unpoled samples has already been reported in a recent paper [9]. García et al. prove that the existence of such an anomaly does not depend on the crystallographic phase, but is related to the presence of oxygen vacancies. The

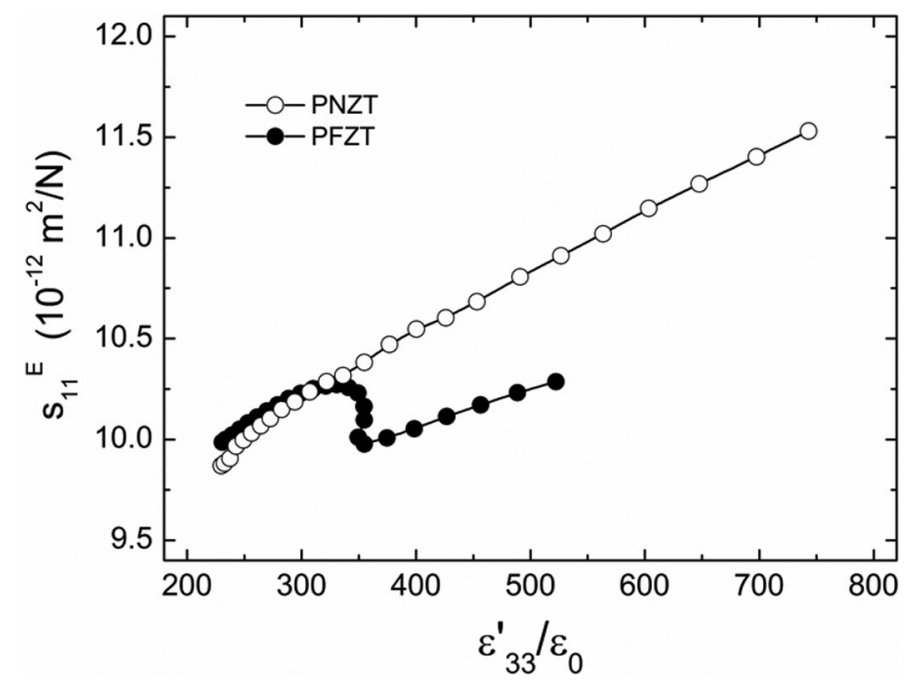

Fig. 4. Compliance $s_{11}{ }^{E}$ versus dielectric constant $\varepsilon^{\prime} / \varepsilon_{0}$ in donor-doped (PNZT) and acceptor-doped (PFZT) PZT ceramics. Significant changes are produced in PFZT behavior.

present results show that this anomaly appears in poled samples over the same temperature range, not only in the dielectric response, but also in the piezoelectric and elastic responses. Furthermore, the dynamics of the domain wall motion of soft PZT is similar over the whole temperature range. This appears to be due to the fact that these materials have a very low concentration of oxygen vacancies [8].

To explain the experimental differences between soft and hard PZT, a reasonable hypothesis is that 2 different mechanisms exist, each having an influence on the extrinsic response. The first one varies directly according to the temperature and is the dominant mechanism in the dielectric response of soft PZT over the whole range. This mechanism corresponds to the domain wall motion in a medium that contains randomly distributed defects (or pinning centers), so that permittivity regularly rises with the temperature [11]. The second mechanism would produce the inhibition of the first under some conditions, starting from the temperature at which the anomaly is observed. Although the nature of this last mechanism is still unknown, the results of this work show that it is related to the presence of complex defects associated with oxygen vacancies produced in hard PZT. It should be pointed out that if one mechanism inhibits the other, it implies that both contributions cannot be added, so that the behavior must be nonlinear. Hence, a change in the dynamics of the domain wall motion can be expected in a region where both mechanisms are sufficiently important.

In summary, the unexpected behavior of the extrinsic effect when hardener dopants are added is the origin of the clear differences between soft and hard PZT at room temperature. The anomaly of the extrinsic response observed in hard PZT is manifested by a decrease in the values of the dielectric, piezoelectric, and elastic coefficients with respect to the soft PZT. However, it is also the cause of the higher thermal stability of hard PZT, as well as the 
noticeable decrease in losses at room temperature. The anomaly is directly related to the addition of acceptor dopants, which enables us to state that such anomaly is related to the formation of complex defects created by the existence of oxygen vacancies.

\section{ACKNOWLEDGMENT}

The authors wish to thank to Prof. J. A. Eiras for useful discussion and for providing the samples.

\section{REFERENCES}

[1] B. Jaffe, W. R. Cook, and H. Jaffe, Piezoelectric Ceramics. London, England: Academic, 1971.

[2] A. J. Moulson and J. M. Herbert, Electroceramics: Materials, Properties, Applications, 2nd ed. Chichester, England: John Wiley \& Sons, 2003.

[3] Q. M. Zhang, H. Wang, N. Kim, and L. E. Cross, "Direct evaluation of domain wall and intrinsic contributions to the dielectric and piezoelectric response and their temperature dependence on lead zirconate titanate ceramics," J. Appl. Phys., vol. 75, pp. 454-459, Jan. 1994.

[4] D. Damjanovic and M. Demartin, "Contribution of the irreversible displacement of domain walls to the piezoelectric effect in barium titanate and lead zirconate titanate ceramics," J. Phys. Condens. Matter, vol. 9, pp. 4943-4953, Jun. 1997.

[5] P. M. Chaplya and G. P. Carman, "Dielectric and piezoelectric response of lead zirconate-lead titanate at high electric and mechanical loads in terms of non- $180^{\circ}$ domain wall motion," J. Appl. Phys., vol. 90, pp. 5278-5286, Nov. 2001.

[6] S. Takahashi, "Effects of impurity doping in lead zirconate-titanate ceramics," Ferroelectrics, vol. 41, no. 1, pp. 277-290, 1982

[7] W. L. Warren, G. E. Pike, K. Vanheusden, D. Dimos, B. A. Tuttle, and J. Robertson, "Defect-dipole alignment and tetragonal strain in ferroelectrics," J. Appl. Phys., vol. 79, pp. 9250-9257, Jun. 1996.

[8] Z. Zhang, L. Lu, C. Shu, and P. Wu, "Computational investigation of B-site donor doping effect on fatigue behavior of lead zirconate titanate," Appl. Phys. Lett., vol. 89, art. no. 152909, 2006.

[9] J. E. García, V. Gomis, R. Pérez, A. Albareda, and J. A. Eiras, "Unexpected dielectric response in lead zirconate titanate ceramics: The role of ferroelectric domain wall pinning effects," Appl. Phys. Lett., vol. 91, art. no. 042902, 2007.

[10] IEEE Standard on Piezoelectricity, ANSI/IEEE std. 176, 1987.

[11] T. Nattermann, Y. Shapir, and I. Vilfan, "Interface pinning and dynamics in random systems," Phys. Rev. B, vol. 42, no. 13, pp. 8577-8586, 1990. 